\title{
BMJ Open Nutritional Outcomes of patients Undergoing Resection for upper gastroIntestinal cancer in AuStralian Hospitals (NOURISH): protocol for a multicentre point prevalence study
}

\author{
Irene Deftereos (D) , ${ }^{1,2}$ Justin M C Yeung, ${ }^{1,3,4}$ Vanessa M Carter, ${ }^{2}$ \\ Elizabeth Isenring, ${ }^{5,6}$ Nicole K Kiss, ${ }^{7,8}$ NOURISH Point Prevalence Study Group
}

To cite: Deftereos I, Yeung JMC, Carter VM, et al. Nutritional Outcomes of patients Undergoing Resection for upper gastrolntestinal cancer in AuStralian Hospitals (NOURISH): protocol for a multicentre point prevalence study. BMJ Open 2020;10:e035824. doi:10.1136/ bmjopen-2019-035824

- Prepublication history and additional material for this paper are available online. To view these files, please visit the journal online (http://dx.doi. org/10.1136/bmjopen-2019035824).

Received 18 November 2019 Revised 22 January 2020 Accepted 14 April 2020

Check for updates

(C) Author(s) (or their employer(s)) 2020. Re-use permitted under CC BY-NC. No commercial re-use. See rights and permissions. Published by BMJ.

For numbered affiliations see end of article.

Correspondence to

Irene Deftereos;

irene.deftereos@unimelb.edu.au

\section{ABSTRACT}

Introduction Nutritional intervention and prevention of malnutrition is significantly important for patients with upper gastrointestinal oesophageal, pancreatic and gastric cancer. However, there is limited information regarding nutritional status, and perioperative nutritional interventions that patients receive when undergoing curative surgery.

Methods and analysis Patients diagnosed with upper gastrointestinal cancer, planned for curative intent resection across 27 Australian hospitals will be eligible to participate in this point prevalence study. The primary aim is to determine the prevalence of malnutrition in patients with upper gastrointestinal cancer at the time of surgery using subjective global assessment. Secondary aims are to determine the type and frequency of perioperative nutritional intervention received, the prevalence of clinically important weight loss and low muscle strength, and to investigate associations between the use of an evidence-based nutrition care pathway or protocol for the nutritional management of upper gastrointestinal surgical oncology patients and malnutrition prevalence. Data collection will be completed using a purpose-built data collection tool.

Ethics and dissemination Ethical approval was granted in May 2019 (LNR/51107/PMCC-2019). The design and reporting of this study comply with the Strengthening the Reporting of Observational Studies in Epidemiology checklist for reporting of observational cohort studies. Findings will be published in peer-reviewed scholarly journals and presented at relevant conferences. Results will assist in defining priority areas for research to improve patient outcomes.

\section{INTRODUCTION}

Patients with oesophageal, gastric and pancreatic cancer, known as upper gastrointestinal (UGI) cancer, can experience significant nutritional issues. Malnutrition is an independent risk factor for increased mortality, surgical complications, length of stay (LOS) and decreased chemo/radiotherapy
Strengths and limitations of this study

- This is the largest study to prospectively conduct nutritional assessment of patients with upper gastrointestinal cancer at the time of surgery, using validated assessment methods.

- The unique dataset will provide a detailed snapshot of nutritional status and associated clinical and service delivery outcomes.

- Limitations of the study include potential variations in data collection procedures; however, all sites will undergo training and follow standardised protocols to ensure reliable results.

tolerance and quality of life (QOL). ${ }^{1-3}$ Rates of malnutrition in patients with UGI cancer are among the highest of all tumour groups, reported to be between $40 \%$ and $80 \%$ at any given time post diagnosis. ${ }^{4-9}$ However, there is limited prospective data regarding the nutritional status of patients with UGI cancer at the time of curative intent surgery using validated assessment methods.

Practice guidelines recommend early and sustained nutritional intervention for patients with cancer who are at high risk of nutritional decline. ${ }^{2}$ In major abdominal surgery, perioperative optimisation is strongly recommended. ${ }^{3}$ However, despite the increasing body of evidence regarding the importance of prevention and treatment of malnutrition, there are no published evidence-based practice guidelines for nutritional intervention in UGI cancers specifically. ${ }^{10}$ Robust evidence to support perioperative nutritional interventions specific to this patient group is not available in the current literature. Subsequently, significant variation in the self-reported nutritional practices of dietitians and surgeons have been previously demonstrated, ${ }^{11} 12$ and 
clinical screening and assessment programmes are often inadequate. ${ }^{13}$ While some research is being undertaken to understand the nutrition support received for patients with head and neck, and oesophageal tumours, ${ }^{14}$ there is a significant lack of information regarding the perioperative nutritional interventions that patients with UGI cancer receive when undergoing curative surgery. Further exploration of current nutritional status, nutritional practices and associated surgical outcomes is required to inform the development of clinical trials to optimise nutrition management, which will ultimately lead to the development of evidence-based practice guidelines. The Nutritional Outcomes of patients Undergoing Resection for upper gastroIntestinal cancer in AuStralian Hospitals (NOURISH) Point Prevalence Study aims to ascertain this important information.

The primary aims are as follows:

1. To determine the prevalence of malnutrition at the time of surgery in patients undergoing resection for UGI cancer using subjective global assessment (SGA).

2. To determine the type and frequency of perioperative dietetics and nutritional intervention received.

Secondary aims are as follows:

1. To determine the prevalence of clinically important weight loss $(>5 \%)$ and low muscle strength from hand grip strength dynamometry.

2. To investigate associations between nutritional status, dietetics intervention and surgical outcomes (LOS, discharge destination, surgical complications).

3. To investigate associations between the use of an evidence-based nutrition care pathway or protocol for the nutritional management of UGI surgical oncology patients and malnutrition prevalence.

\section{METHODS}

\section{Study design and setting}

This is an observational point prevalence study that will be conducted at 27 tertiary metropolitan hospitals across Victoria, New South Wales, Queensland, South Australia, Western Australia and Australian Capital Territory, Australia (participating sites can be viewed in online supplementary file 1 ). The minimum recruitment period will be 4 months (or minimum 10 participants per site), representing at least one-third of the yearly UGI cancer cohort undergoing surgery at each site. Recruitment commenced on the 2 September 2019, and data collection will be completed by 30 June 2020 .

\section{Inclusion and exclusion criteria}

Patients will be considered eligible for participation if they are 18 years or older and are inpatients after curative intent UGI cancer surgery, including those who have received or not received neoadjuvant therapy. Eligible surgery types include gastrectomy (subtotal, total, partial), pancreatectomy (total, distal, partial, pancreaticoduodenectomy), oesophagectomy or gastro-oesophagectomy. Patients must be able to consent to participation via
English language communication, or with the presence of an interpreter. Patients will be excluded if they underwent palliative surgery, are unable to provide consent or participate in assessment, are taking intravenous opioids at the time of consent, are unaware of their diagnosis of malignancy, or assessment of nutritional status does not occur within 7 days of surgery.

\section{Outcome measurements and data collection}

Figure 1 outlines the study data collection schedule. Site investigators will conduct a nutritional assessment and survey of the participants at a single timepoint within 7 days of their UGI cancer surgery, to accurately reflect nutritional status at the time of surgery. Data will also be collected from the medical record during the participant's hospital admission, which will include information regarding dietetics intervention, nutritional intake and surgical outcomes. All site investigators are experienced clinical dietitians who are trained in completion of all associated components of nutrition assessment. Furthermore, all site investigators will receive training by the research team via webinar prior to study commencement to ensure standard practices and reliability in data collection and entry across sites, as per previous large prevalence studies. ${ }^{8}$ Site principal investigators (PI) will also participate in a questionnaire pertaining to site-specific practices for the nutritional management of UGI surgical oncology patients. ${ }^{15} 16$ The data collection tool can be viewed in online supplementary file 2 . Table 1 outlines the methods and modes of data collection for patient data.

\section{Demographic characteristics}

Baseline demographic characteristics will include age, sex, primary language spoken, usual social situation, distance from hospital and if identified from a Culturally and Linguistically Diverse or Aboriginal and Torres Strait Islander background.

\section{Tumour and surgical characteristics}

Tumour characteristics will include date of diagnosis, tumour site, type and pathological staging from recorded biopsy results at time of surgery, as per defined tumour node metastasis staging system. ${ }^{17}$ Details regarding neoadjuvant chemo/radiotherapy receipt and completion will also be documented. The surgical procedure details including date, type and surgical technique used will also be recorded.

\section{Nutritional status}

\section{Anthropometry and body mass index}

Weight will be measured using standard hospital weighing equipment on the day of nutritional assessment within 7 days of surgery, and within 2 days prior to hospital discharge. Investigators will also determine the presence of oedema and will record estimated dry weight if oedema is present. If patients are unable to be weighed within 7 days of surgery, they will be asked to recall their weight from the week prior to surgery. Patients will also be asked 
PATIENT DATA

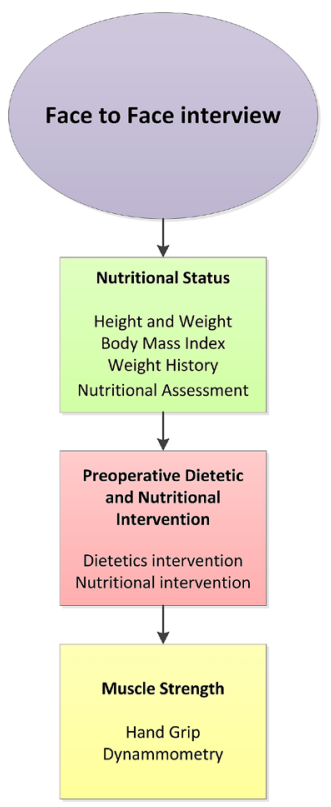

Figure 1 Study data collection schedule.

to recall and report their weight history from 2 weeks, $1,3,6$ and $>6$ months prior to their surgery. Weight will be recorded to the nearest $0.5 \mathrm{~kg}$, and percentage (\%) weight loss will be calculated to the nearest $0.1 \%$ for each timepoint. Percentage weight loss will be used as part of the nutritional assessment, as well as to report on the prevalence of clinically important weight loss $>5 \%$, as per secondary aims. Height will be measured using a wallmounted stadiometer or can be patient reported, to the nearest centimetre. Patient reported weight and height history has been demonstrated to be reliable against actual history. ${ }^{18}$ If patients cannot recall their weight or height and are unable to be measured, the medical history will be checked for this information, and if unavailable it will be marked as missing data. Body mass index (BMI) will be calculated using the following formula: BMI $(\mathrm{kg} /$ $\mathrm{m}^{2}$ )=weight $(\mathrm{kg}) /$ height squared $\left(\mathrm{m}^{2}\right) .{ }^{19}$ BMI will be used to assess if patient fulfils the international definition of malnutrition, as specified below.

\section{Assessment of nutritional status}

Nutritional status will be evaluated using the SGA, which has been validated as a nutritional assessment tool in hospitalised populations, including oncology patients. ${ }^{20}$

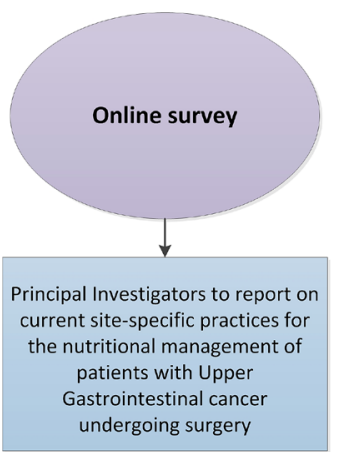

It consists of: (1) historical components: weight history, dietary intake changes, gastrointestinal symptom history and reported activities and function. (2) Physical assessment: muscle and fat loss, and fluid accumulation.

The clinician provides an overall subjective assessment rating, based on the above SGA components: (1) well nourished, (2) mild/moderate or suspected malnutrition or (3) severe malnutrition. A standardised protocol will be adopted for completion of the SGA across all sites. Further details of the components of the SGA are outlined in the data collection tool (online supplementary file 2).

The dietitian will also assess for malnutrition using The International Classification of Diseases, 10th Revision Australian modification (ICD-10- AM) classification for malnutrition, to ensure that the appropriate dietetics intervention is provided in the clinical setting per Australian malnutrition coding procedures. ${ }^{21}$ Based on the components of the SGA, ICD-10-AM defines malnutrition as $\mathrm{BMI}<18.5 \mathrm{~kg} / \mathrm{m}^{2}$ or unintentional loss of weight (5\%-9\%) with evidence of suboptimal intake resulting in moderate loss of subcutaneous fat and/or moderate muscle wasting. 
Table 1 Methods and modes of patient data collection

\begin{tabular}{lll} 
Outcome measurement & $\begin{array}{l}\text { Face to face interview } \\
\text { with patient }\end{array}$ & $\begin{array}{l}\text { Data collection from } \\
\text { medical records }\end{array}$ \\
\hline Demographic, tumour and surgical characteristics & $\mathrm{X}$ & $\mathrm{X}$ \\
$\begin{array}{l}\text { Nutritional status } \\
\text { Subjective global assessment }\end{array}$ & $\mathrm{X}$ \\
$\begin{array}{l}\text { International Classification of Diseases Australian Modification Malnutrition } \\
\text { Diagnosis }\end{array}$ & $\mathrm{X}$ & \\
\hline Height & $\mathrm{X}$ & $\mathrm{X}^{*}$ \\
\hline Weight (baseline and end of hospital admission) & $\mathrm{X}$ & $\mathrm{X}^{*}$ \\
\hline Body mass index & $\mathrm{X}$ & \\
\hline Weight history & $\mathrm{X}$ & $\mathrm{X}^{*}$ \\
\hline Muscle strength using hand grip strength dynamometer & $\mathrm{X}$ & \\
\hline Dietetics and nutritional intervention & & $\mathrm{X}$ \\
\hline Inpatient dietetics intervention & & $\mathrm{X}$ \\
\hline Prior dietetics intervention & $\mathrm{X}$ & $\mathrm{X}$ \\
\hline Postdischarge dietetics intervention & & $\mathrm{X}$ \\
\hline Prior nutritional intervention & $\mathrm{X}$ & $\mathrm{X}$ \\
\hline Nutritional intake during hospital admission & & \\
\hline Surgical outcomes and length of stay & & \\
\hline
\end{tabular}

${ }^{*}$ Check baseline information postpatient interview if required.

\section{Dietetics intervention}

Prior dietetics intervention

Participants will be asked to recall if they have seen a dietitian prior to surgery, since their cancer diagnosis. If patients have seen a dietitian during this time, the location and timing of the last contact with the dietitian will be recorded if known.

\section{Inpatient dietetics intervention}

The day of first dietetics contact with the patient postoperatively will be recorded. Dietetics intervention provided for the first 10 days following surgery will be documented, including dietary counselling, enteral nutrition (EN), parenteral nutrition (PN), oral nutrition support (ONS) or no nutrition intervention. The hospital 'diet codes' of Nil by Mouth, Clear Fluids, Free Fluids, Pureed or Minced, Light ward diet, Full Ward Diet, Soft Ward Diet will also be recorded. At the end of the patient's inpatient admission the total number of contacts by the dietitian will be calculated.

\section{Postdischarge dietetics intervention}

Dietetics follow-up arrangements for each patient on discharge will be documented including timeframe for follow-up (within 2 weeks; $2-4$ weeks; $4-8$ weeks; >8 weeks) and location of service (on site specialist UGI clinic; on site general clinic; community health service dietitian; private dietitian). The hospital 'diet code' on discharge, and the provision of ONS and EN will be documented, including the percentage of estimated energy and protein requirements met by ONS and EN.

\section{Nutritional intervention}

Participants will be asked to recall if they have received any preoperative nutritional advice from their surgeon, oncologist, general practitioner or other healthcare professional; and what advice was provided. Participants will also be asked to recall if they have been receiving any nutritional support including ONS drinks, immunonutrition drinks, carbohydrate loading drinks, EN or PN prior to surgery.

\section{Nutritional intake}

Patients will be asked to recall any decreases in their food intake before surgery by estimating the decrease compared with normal, recorded as $\geq 75 \%, \leq 75 \%, \leq 50 \%$, $\leq 25 \%$ of their usual intake. Postoperative estimated energy requirements (EER) and estimated protein requirements (EPR) at the time of surgery will be determined by the site dietitian using prediction equations. The proportion of EER and EPR met since last dietitian review (ranges $<20 \%, 20 \%-40 \%, 40 \%-60 \%, 60 \%-80 \%$, $80 \%-100 \%$ ), as calculated by the dietitian by determining daily intake against EER and EPR during clinical reviews will be documented for the first 10 days postoperatively. This will provide information regarding compliance and adequacy of nutrition intervention prescribed.

\section{Muscle strength}

Low muscle mass is a key component of malnutrition diagnosis, and a strong determinant of outcomes in patients with cancer undergoing surgery. ${ }^{22}$ The use of pre-existing diagnostic CT scans to determine skeletal muscle index 
in oncology patients, at the level of lumbar spine 3 (L3) is well validated. However, due to resource constraints of this study with 27 recruitment sites, we are not able to perform objective analysis of muscle mass using CT or other measures. Muscle strength measurement can be used as a surrogate measurement of muscle mass, in the absence of an objective measure. ${ }^{22}$ Muscle strength will be measured using hand dynamometers as per the methodology of the American Society of Hand Therapists (ASHT), which is considered the gold standard. ${ }^{23}$ Sites will be provided with a training package and a standard protocol to follow ASHT methodology, to ensure reliability. Assessment of hand grip strength by different assessors can be considered interchangeable, if assessors follow the same protocol. ${ }^{23}$ The Australian and New Zealand Society for Sarcopenia and Frailty Research and the newly released Global Leadership Initiative on Malnutrition criteria suggested muscle strength cut-off values of $<20 \mathrm{~kg}$ for women and $<30 \mathrm{~kg}$ for men for the diagnosis of sarcopenia. ${ }^{22}$ The Asian Working Group for Sarcopenia adapted cut-off values of $<18 \mathrm{~kg}$ for women and $<26 \mathrm{~kg}$ for men will be used for participants of Asian background. ${ }^{24}$ The mean value of both left and right hands will be recorded, and the highest value will be used for muscle function analysis as per the ASHT methodology.

\section{Surgical outcomes and LOS}

LOS will be calculated once the patient is discharged from the acute surgical admission. Discharge destination (home, aged care facility, inpatient rehabilitation, death) will be recorded. Incidence of surgical complications known to be impacted by malnutrition will be recorded from the medical file as documented by the surgical team, including presence of surgical site/wound infection, sepsis, anastomotic leak, fistula, respiratory tract infection, pneumonia, pressure injury, wound dehiscence, gastroparesis, chyle leak, return to theatre, abdominal collection or ileus.

\section{Site Specific Dietetics Practices Questionnaire}

Site PI dietitians will be invited to participate in a purpose built, online questionnaire at the commencement of the study on behalf of their site. Information regarding current site-specific practices for the nutritional management of UGI surgical oncology patients, including the use of evidence-based protocols or pathways such as Enhanced Recovery After Surgery (ERAS), and provision of inpatient and outpatient perioperative dietetics services will be collected. Results of the questionnaire will be linked with patient data to investigate associations to address the secondary aims of the study. The site questionnaire can be viewed as online supplementary file 3 .

\section{Statistical considerations}

Estimated sample size

Sample size is pragmatic based on patients presenting to the sites within the above timeframes in $2017 / 2018$. Total recruitment sample size is expected to be a minimum of
200 participants. Assuming malnutrition prevalence is $50 \%$, the corresponding $95 \% \mathrm{CI}$ is $(43 \%$ to $57 \%)$, that is, a precision of $\pm 7 \%$, which is considered appropriate for the primary outcome. Achieved precision will be higher if the prevalence is closer to $0 \%$ or to $100 \%$.

\section{Statistical analysis}

The primary aim, estimating the proportion of patients with malnutrition, will be obtained from the observed point prevalence with exact 95\% CIs. Descriptive statistics will be used to describe baseline characteristics of participants, nutritional status measurements and nutritional intervention received. For normally distributed data, means and SDs will be used; with medians and IQRs for non-normally distributed data. Counts and percentages will also be used as appropriate. Associations between nutritional status, use of evidence-based nutrition care pathways/procedures and surgical outcomes will be investigated using multiple logistic regression analysis, and will also account for tumour type and stage (1-4), age and sex. LOS will be treated as time to event and analysed univariately with Kaplan-Meier and the log-rank test. Subsequently a Cox proportional hazard analysis will be used to adjust for tumour type and stage, age and sex. The proportional hazards assumption will be assessed by a global test of the residuals. Responses to the site-specific practices questionnaire will be presented descriptively using means and SDs or medians and IQR and counts and percentages as appropriate to the format of each question. Free text responses will be summarised. The statistical package SPSS (V.24 or newest release (licensed to the University of Melbourne, Chicago, Illinois, USA)) will be used for quantitative data analysis.

\section{Patient and public involvement}

Patients/the public were not involved in the design of this study, however patient feedback from a previous study was used to design the data collection tool. Each patient will be made aware of their individual nutrition assessment. The high number of sites and participants makes it impractical to provide group results to patients individually.

\section{ETHICS AND DISSEMINATION Approval}

This project received full ethical approval under the Australian National Mutual Acceptance ethical review scheme by The Peter MacCallum Cancer Centre Ethics Committee in May 2019 (LNR/51107/PMCC-2019). Ethics and governance approval at each participating hospital will also be obtained prior to commencement at each site (online supplementary file 1). The study will be conducted as per the National Health and Medical Research Council (NHMRC) National Statement on Ethical Conduct in Human Research (2007 and updates) and the World Medical Association Declaration of Helsinki (2013 and updates). The design and reporting of 
this study complies with the Strengthening the Reporting of Observational Studies in Epidemiology checklist for reporting of observational cohort studies. ${ }^{25}$

\section{Informed consent}

Study dietitians will identify and approach eligible patients prior to, or at the time of surgical admission by screening surgical procedure lists and liaising with surgical and nursing staff. Patients will be provided with a study patient information statement (PIS), and will be given a minimum of 24 hours to read it. Study dietitians will also explain the study verbally and answer questions. The patient will then be asked to provide verbal consent for participation, once they can confirm that they have read and understood the PIS and what their participation will involve. Consent will be obtained and documented in accordance with international good clinical practice (GCP) requirements, and all study personnel will undergo appropriate training. Patients will be asked to re-confirm verbal consent prior to the physical assessment component of the SGA, the hand grip strength test, as well as re-confirm consent for data collection from their medical record. Participants will also be informed that they may withdraw their consent at any time. Both participants and non-participants will receive standard dietetics care during the study.

\section{Data collection and management}

Study data will be collected and managed via a purposebuilt, secure electronic data collection tool (REDCap, hosted at The University of Melbourne), which will be used for data collection and entry. ${ }^{15} 16$ Participant data will be anonymised using unique study identification numbers prior to data entry into the REDCap database. Only the coordinating researcher (ID) will have access to the full de-identified dataset, and each site will have access to their own data. If paper copies of the data collection form are used, they will also be anonymised, and stored in the locked offices of the dietetics department at each site. The principal investigator at each site will be responsible for the management and security of data. The site-specific practices questionnaire will be completed online only via the project's secure REDCap survey link by the principal investigator at each site. Data will be kept for 5 years from the completion of the project, in accordance with the NHMRC National Statement on Ethical Conduct in Human Research (2007 and updates) and international GCP requirements.

\section{Results dissemination}

Findings from this study to be published in peer-reviewed scholarly journals and presented at relevant conferences national and internationally. Any published data will be aggregated and de-identified. The study is being undertaken in part-fulfilment of the Doctor of Philosophy $(\mathrm{PhD})$ of the coordinating researcher (ID) at The University of Melbourne, Melbourne, Australia.

\section{DISCUSSION}

Malnutrition is a significant problem in UGI cancer, with up to $80 \%$ of patients reporting more than $5 \%$ or $10 \%$ wt loss at initial presentation. ${ }^{96} 27$ Nutrition support should be commenced as early as possible and continued throughout the perioperative period. ${ }^{3}$ However, there is limited evidence regarding the optimal methods of nutritional intervention, leading to inconsistencies in clinical practice by members of the multidisciplinary team, including dietitians. ${ }^{11-13} 28$

Further research is required to determine the most effective intervention methods to optimise nutrition, QOL and surgical outcomes, to develop evidence-based practice guidelines. The relatively low incidence of UGI cancer amenable to curative intent surgery makes it difficult to conduct high quality, effective nutritional research, and this could be one of the reasons why clinical nutrition trials in this high-risk patient group are significantly lacking in Australia and internationally. A thorough understanding of the nutritional needs of the UGI surgical oncology population and current practices in relation to current best available evidence is required to inform the development of clinical trials, and for the development of evidence-based practice guidelines. ${ }^{10}$ However, current data regarding the prevalence of malnutrition, perioperative nutrition and dietetics practices, and clinical outcomes is inadequate. The results of this study will inform the current evidence base in UGI surgical oncology nutrition by providing a robust cross-sectional dataset of malnutrition prevalence rates obtained using validated assessment methods, and a unique detailed understanding of the perioperative dietetics and nutritional intervention received by patients across 27 Australian tertiary hospitals. The results of this study will identify priority areas for the design of prospective research and quality improvement, ultimately aiming to improve the outcomes of patients undergoing resection for UGI cancer.

\section{Author affiliations}

${ }^{1}$ Department of Surgery Western Health, The University of Melbourne, Melbourne, Victoria, Australia

${ }^{2}$ Nutrition and Dietetics, Western Health, Footscray, Victoria, Australia

${ }^{3}$ Department of Colorectal Surgery, Western Health, Footscray, Victoria, Australia ${ }^{4}$ Western Chronic Disease Alliance, Western Health, Melbourne, Victoria, Australia ${ }^{5}$ Faculty of Health Sciences and Medicine, Bond University, Robina, Queensland, Australia

${ }^{6}$ Department of Nutrition and Dietetics, Princess Alexandra Hospital, Brisbane, Queensland, Australia

${ }^{7}$ Institute for Physical Activity and Nutrition, Deakin University, Geelong, Victoria, Australia

${ }^{8}$ Department of Cancer Experiences Research, Peter MacCallum Cancer Centre, Melbourne, Victoria, Australia

Twitter Irene Deftereos @IreneDeftereos and NOURISH Point Prevalence Study Group @NOURISH_UpperGl

Acknowledgements The authors wish to thank Dr Koen Simons (Office for Research, Western Health and The School of Population Health, The University of Melbourne) for his advice regarding statistical analysis. The lead researchers would also like to thank all participating study dietitians for generously providing their time to undertake this study, and their managers for providing their support of the study. 
Collaborators Members of the NOURISH Point Prevalence Study Group: Anna Cardamis (Eastern Health, Melbourne), Annika Dorey (Cabrini Hospital, Melbourne), Aurora Ottaway (Western Health, Melbourne), Brook Maguire (Westmead Hospital, Sydney), Brydie Cleeve (Epworth HealthCare, Melbourne), Caitlin Davis (Flinders Medical Centre, Adelaide), Christine Choong (Austin Health, Melbourne), Claire Douglas (Prince of Wales Hospital, Sydney), Claire Nixon (The Royal Adelaide Hospital, Adelaide), Daniel Platt (Cabrini Hospital, Melbourne) Eleanor Quinn (John Hunter Hospital, Newcastle), Eliza Simpson (The Royal Adelaide Hospital, Adelaide), Emma Hamdorf (The Queen Elizabeth Hospital, Adelaide), Emma McNamara (John Hunter Hospital, Newcastle), Emma Whelan (Sir Charles Gairdner Hospital, Perth), Gayathri Jegendran (Liverpool Hospital, Sydney), Georgia Moore (Eastern Health, Melbourne), Georgina Lockwood (Sunshine Coast Hospital and Health Service, Sunshine Coast), Jacqueline McNamara (Western Health, Melbourne), Jemma Corrigan (Canberra Hospital, Canberra), Kate Fox (St Vincent's Hospital Melbourne, Melbourne), Kate Furness (Monash Health, Melbourne), Kiah Witney Cochrane (Royal Prince Alfred Hospital, Sydney), Kieu Huynh (Bankstown Lidcombe Hospital, Sydney), Nadia Hames (Westmead Hospital, Sydney), Nadia Hendricks (St Vincent's Hospital Melbourne, Melbourne), Naomi Page (St George Hospital, Sydney), Natalie Brooks (Fiona Stanley Hospital, Perth), Lauren Nevin (Barwon Health), Lindy Parfrey (The Queen Elizabeth Hospital, Adelaide), Emma Putrus (The Royal Adelaide Hospital, Adelaide), Rachel Pons (St George Hospital, Sydney), Roy Hoevenaars (Barwon Health, Geelong), Sheena Singh (Concord Hospital, Sydney), Simone McCoy (Princess Alexandra Hospital, Brisbane), Siobhan Wallin (The Royal Brisbane and Women's Hospital, Brisbane), Stella Mexias (Northern Health, Melbourne), Suzie Daniells (Prince of Wales Hospital, Sydney), Tayla Storr (The Townsville Hospital and Health Service, Townsville), Tayla Robertson (Princess Alexandra Hospital, Brisbane), Teresa Brown (The Royal Brisbane and Women's Hospital, Brisbane).

Contributors ID is the coordinating investigator and conceptualised the study. NKK, JMCY, El and VMC contributed to the study conception and design. ID drafted and edited the study protocol and manuscript. NKK, JMCY, VMC and El assisted with the study protocol and edited the manuscript. Members of the NOURISH Point Prevalence Study Group provided feedback on data collection procedures and will carry out the study recruitment and data collection. All authors have read and approved the final version of the manuscript.

Funding An unrestricted project grant has been received from Nestlé Health Science to support out of pocket expenses for the operation of this study. The funder was not involved in the study design, and will not be involved in results analysis, interpretation or dissemination.

Competing interests None declared.

Patient consent for publication Not required.

Provenance and peer review Not commissioned; externally peer reviewed.

Open access This is an open access article distributed in accordance with the Creative Commons Attribution Non Commercial (CC BY-NC 4.0) license, which permits others to distribute, remix, adapt, build upon this work non-commercially, and license their derivative works on different terms, provided the original work is properly cited, appropriate credit is given, any changes made indicated, and the use is non-commercial. See: http://creativecommons.org/licenses/by-nc/4.0/.

\section{ORCID iD}

Irene Deftereos http://orcid.org/0000-0002-3822-147X

\section{REFERENCES}

1 Arends J, Baracos V, Bertz H, et al. ESPEN expert group recommendations for action against cancer-related malnutrition. Clin Nutr 2017;36:1187-96.

2 Arends J, Bachmann P, Baracos V, et al. ESPEN guidelines on nutrition in cancer patients. Clin Nutr 2017;36:11-48.

3 Weimann A, Braga M, Carli F, et al. ESPEN guideline: clinical nutrition in surgery. Clin Nutr 2017;36:623-50.

4 Baldwin C, McGough C, Norman AR, et al. Failure of dietetic referral in patients with gastrointestinal cancer and weight loss. Eur $J$ Cancer 2006;42:2504-9.

5 Bozzetti F, Mariani L, Lo Vullo S, et al. The nutritional risk in oncology: a study of 1,453 cancer outpatients. Support Care Cancer 2012;20:1919-28.
6 Senesse P, Assenat E, Schneider S, et al. Nutritional support during oncologic treatment of patients with gastrointestinal cancer: who could benefit? Cancer Treat Rev 2008;34:568-75.

7 Grace EM, Shaw C, Lalji A, et al. Nutritional status, the development and persistence of malnutrition and dietary intake in oesophagogastric cancer: a longitudinal cohort study. J Hum Nutr Diet 2018;31:785-92

8 Marshall KM, Loeliger J, Nolte L, et al. Prevalence of malnutrition and impact on clinical outcomes in cancer services: a comparison of two time points. Clin Nutr 2019;38:644-51.

9 La Torre M, Ziparo V, Nigri G, et al. Malnutrition and pancreatic surgery: prevalence and outcomes. J Surg Oncol 2013;107:702-8.

10 Lu Y, Carey S. Translating evidence-based practice guidelines into a summary of recommendations for the nutrition management of upper gastrointestinal cancers. Nutr Clin Pract 2014;29:518-25.

11 Lahoud J, Bazzi K, Yeo D, et al. Survey of nutritional practices in total gastrectomy and oesophagectomy procedures. Nutr Diet 2019;76:135-40.

12 Carey S, He L, Ferrie S. Nutritional management of patients undergoing major upper gastrointestinal surgery: a survey of current practice in Australia. Nutr Diet 2010;67:219-23.

13 Williams JD, Wischmeyer PE. Assessment of perioperative nutrition practices and attitudes-A national survey of colorectal and Gi surgical oncology programs. Am J Surg 2017;213:1010-8.

14 ClinicalTrials.gov. National Library of Medicine (U.S). International Nutrition Audit in FORegut TuMors (INFORM)- Identifier NCT0289489, 2016. Available: https://ClinicalTrials.gov/show/ NCT02829489 [Accessed Nov 2019].

15 Harris PA, Taylor R, Minor BL, et al. The REDCap Consortium: building an international community of software platform partners. $J$ Biomed Inform 2019;95:103208.

16 Harris PA, Taylor R, Thielke R, et al. Research electronic data capture (REDCap)--a metadata-driven methodology and workflow process for providing translational research informatics support. J Biomed Inform 2009;42:377-81.

17 Brody T. Clinical trials: study design, endpoints and biomarkers, drug safety, and FDA and ICH guidelines. St. Louis, United States: Elsevier Science \& Technology, 2011.

18 Haverkort EB, de Haan RJ, Binnekade JM, et al. Self-Reporting of height and weight: valid and reliable identification of malnutrition in preoperative patients. Am J Surg 2012;203:700-7.

19 World Health Organisation (WHO). The International classification of adult underweight, overweight and obesity according to BMI last update, 2019. Available: http://apps.who.int/bmi/index.jsp? introPage=intro_3.html

20 da Silva Fink J, Daniel de Mello P, Daniel de Mello E. Subjective global assessment of nutritional status - a systematic review of the literature. Clin Nutr 2015;34:785-92.

21 Innes K, Hooper J, Bramley M, et al. Creation of a clinical classification. International statistical classification of diseases and related health problems--10th revision, Australian modification (ICD10-AM). Health Inf Manag 1997;27:31-8.

22 Jensen GL, Cederholm T, Correia MITD, et al. GLIM criteria for the diagnosis of malnutrition: a consensus report from the global clinical nutrition community. JPEN J Parenter Enteral Nutr 2019;43:32-40.

23 Roberts HC, Denison HJ, Martin HJ, et al. A review of the measurement of grip strength in clinical and epidemiological studies: towards a standardised approach. Age Ageing 2011;40:423-9.

24 Chen L-K, Liu L-K, Woo J, et al. Sarcopenia in Asia: consensus report of the Asian Working group for sarcopenia. J Am Med Dir Assoc 2014;15:95-101.

25 von Elm E, Altman DG, Egger M, et al. The strengthening the reporting of observational studies in epidemiology (STROBE) statement: guidelines for reporting observational studies. Ann Intern Med 2007;147:573-7.

26 Riccardi D, Allen K. Nutritional management of patients with esophageal and esophagogastric junction cancer. Cancer Control 1999;6:64-72.

27 Ryan AM, Rowley SP, Healy LA, et al. Post-oesophagectomy early enteral nutrition via a needle catheter jejunostomy: 8-year experience at a specialist unit. Clin Nutr 2006;25:386-93.

28 Huddy JR, Huddy FMS, Markar SR, et al. Nutritional optimization during neoadjuvant therapy prior to surgical resection of esophageal cancer-a narrative review. Dis Esophagus 2018;31:1-11. 